\title{
Thrombotic thrombocytopenic purpura (TPP) successfully rescued by plasma exchange in the ICU: A report of two cases
}

\author{
XIULI ZOU, TIEJUN WU, XIHONG ZHANG, AIJUN QU and SUOCHEN TIAN \\ Intensive Care Unit, Liaocheng People's Hospital, Liaocheng, Shandong 252000, P.R. China
}

Received November 25, 2014; Accepted December 22, 2015

DOI: 10.3892/etm.2016.3265

\begin{abstract}
Thrombotic thrombocytopenic purpura (TTP) is a rare, life-threatening disorder, which is characterized by thrombus formation in small blood vessels. The present study retrospectively analyzed the clinical data from two patients with severe TTP, who were treated successfully in the intensive care unit (ICU) at the Liaocheng People's Hospital in 2013. Comprehensive therapies were administered to the patients, including plasma exchange (PE), mechanical ventilation (case 1 only), steroid therapy, blood transfusion and anti-inflammatory treatment (case 2 only). The two patients returned to a stable state and were transferred back to the hematology department following PE. The positive outcome achieved for these patients suggests that early intervention involving bedside PE in the ICU may reduce the mortality rate of patients with severe TTP who have concurrent respiratory or circulatory failure and cannot be treated in the dialysis unit.
\end{abstract}

\section{Introduction}

Thrombotic thrombocytopenic purpura (TTP), which is a rare life-threatening disorder characterized by thrombus formation in small blood vessels, has an incidence of $\sim 37,000$ per 10 million people and is associated with a high mortality rate (1). The signs and symptoms of TTP are purple-colored bruises on the skin or mucous membranes, small-sized red or purple dots on the skin, fatigue, fever, increased heart rate or shortness of breath, headache, speech changes, confusion, coma, stroke or seizure, and low levels of protein in the urine. The main cause of this disease is low activity levels of the ADAMTS13 enzyme and the low expression levels of the ADAMTS13 gene, leading to blood clotting (2).

The most common treatment option is plasma exchange, although other treatment options include medication and

Correspondence to: Dr Tiejun Wu, Intensive Care Unit, Liaocheng People's Hospital, 67 West Dongchang Road, Liaocheng, Shandong 252000, P.R. China

E-mail: tiejunwu@hotmail.com; lcicu@sina.com

Key words: thrombotic thrombocytopenic purpura, blood transfusion, plasma exchange, anti-inflammatory treatment surgical procedures, or occasionally both. The treatments are mostly carried out in clinical settings. Plasma therapy is started immediately following diagnosis of TTP. The frozen plasma is administered through an intravenous injection in order for the inherited TTP to replace the missing or altered ADAMTS13 enzyme. The plasma exchange can also be used for acquired TTP as a life-saving procedure (3). The plasma removes antibodies from the blood that damage the ADAMTS13 enzyme or replaces the ADAMTS13 enzyme. A cell separator is used, which removes plasma from the blood. The non-plasma fraction of the blood is collected, and the donated plasma is added. The blood is then placed into the patient via intravenous administration. This procedure requires $\sim 2 \mathrm{~h}$ to complete. The treatment is then continued until the signs and symptoms of TTP improve. This may take days or weeks, depending on the condition of the patient, and long-term hospitalization may be required for complete recovery. Occasionally, a return of the symptoms may occur either in hospital or when the patient has returned home. In these cases plasma therapy is continued (4-7).

Alternative treatments are not always effective. For acquired TTP, the medications used to treat TTP include glucocorticoids, vincristine, rituximab and cyclosporine A. Surgical removal of the spleen may occasionally be required as the spleen is responsible for the formation of the antibodies that inhibit ADAMTS13 enzyme activity (8).

Previous experimental studies and clinical observations have implicated thrombin sensitive protein 1 (TSP1), anti-endothelial cell antibodies (AECAs) and the excessive release of von Willebrand factor (vWF) multimers in the pathogenesis of TTP (8-11). Since the introduction of plasma exchange (PE) in 1970 s, the mortality rate associated with TTP has gradually decreased from 90 to $10-20 \%$ (12). The present case reports describes two patients with TTP who underwent PE in an intensive care unit (ICU) in 2013 and recovered successfully.

\section{Case reports}

Case 1. A 56-year-old woman was admitted to the Liaocheng People's Hospital (Liaocheng, China) on July 9, 2013 with fatigue, a 7-day history of skin mucosal petechiae, and a 5-h history of convulsions with delirium. A physical examination revealed that the patient had a body temperature of $38.5^{\circ} \mathrm{C}$, a heart rate of 108 beats $/ \mathrm{min}$, a respiratory rate of $23 \mathrm{breaths} / \mathrm{min}$, and a blood pressure of $117 / 96 \mathrm{mmHg}$. At the 


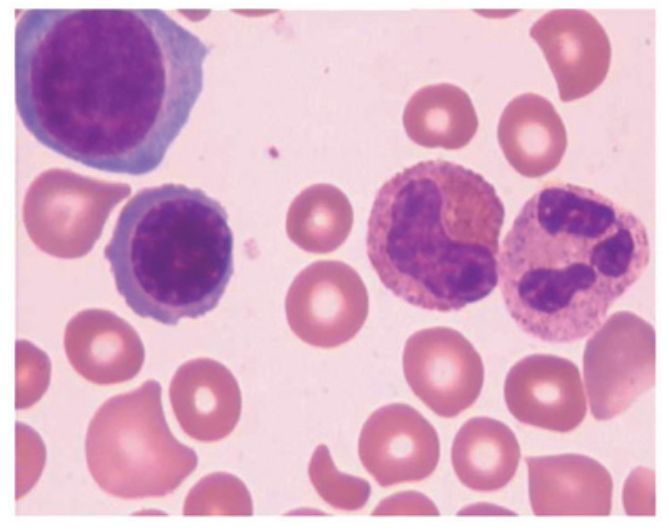

Figure 1. Case 1 bone marrow smear. Bone marrow was stained with Wright Giemsa (magnification, $\mathrm{x} 1,000$ ) demonstrating the presence of abnormally formed erythrocytes.

time of admission, the patient was in a coma (Glasgow Coma Scale, 5) with multiple ecchymoses/petechiae in the skin mucosal membrane over the entire body. Blood tests on admission revealed the following: Hemoglobin $(\mathrm{Hb})$ level, $5.5 \mathrm{~g} / \mathrm{dl}$; platelet (PLT) count, $13 \times 10^{\%} / 1$; total bilirubin, $46 \mu \mathrm{mol} / 1$; and indirect bilirubin, $45 \mu \mathrm{mol} / 1$. No evident abnormality was observed by craniocerebral computed tomography (CT; 64 slice CT scanner, Philips Healthcare, Andover, MA, USA).

A bone marrow biopsy on July 10 indicated a diagnosis of TTP and hemolytic-uremic syndrome (Figs. 1 and 2). The patient's urine tested positive for protein, with $5 \%$ deformed red blood cells. The patient was diagnosed with TTP and multiple organ dysfunction syndrome (MODS), affecting the central nervous, respiratory and circulation systems. Treatment of the patient with PE was recommended; however, it was rejected by the family of the patient due to the high cost. On July 11, tracheal intubation and mechanical ventilation was administered due to frequent convulsions and respiratory failure.

On July 15, the family provided consent and PE by membrane plasma separation (MPS) was performed once daily with a blood flow rate of $120 \mathrm{ml} / \mathrm{min}$ and a plasma extraction rate of $24 \mathrm{ml} / \mathrm{min}$ using Plasma Flux P2 dry plasma exchange filters (Fresenius Medical Care Deutschland GmbH, Wendel, Germany). A total of $2,000 \mathrm{ml}$ plasma was replaced. On July 16, the patient regained consciousness and the mechanical ventilation was removed. Blood tests revealed that the patient's $\mathrm{Hb}$ level was $5.9 \mathrm{~g} / \mathrm{dl}$ and PLT count was $10 \times 10^{9} / 1$. On July 17, a B-mode ultrasound system (Logiq E9; GE Heathcare Life Sciences, Shanghai, China) detected thrombosis formation in the veins of the muscles in the right lower extremity. On July 20, the coagulation mechanism of the patient had been restored to normal with a $\mathrm{Hb}$ level of $6.4 \mathrm{~g} / \mathrm{dl}$ and a PLT count of $41 \times 10^{9} / 1$.

Based on the relatively stable condition of the patient, $\mathrm{PE}$ was performed daily in the hemodialysis room between July 25 and August 7, after which PE treatment was ceased, since the patient had regained a good mental condition and all indices had been restored to within their normal ranges. The patient was discharged from the hospital on August 27, 2013. At the follow-up on September 30, 2013 the patient exhibited no reoccurrence with $\mathrm{Hb}$ levels of $12.4 \mathrm{~g} / \mathrm{dl}$ and PLT counts of $230 \times 10^{9} / 1$.

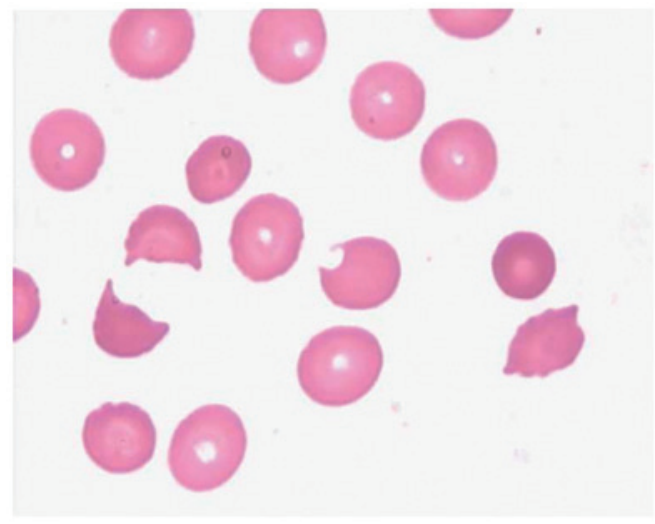

Figure 2. Case 1 peripheral blood smear. Peripheral blood was stained with Wright Giemsa (magnification, x1,000) demonstrating the presence of abnormally formed erythrocytes.

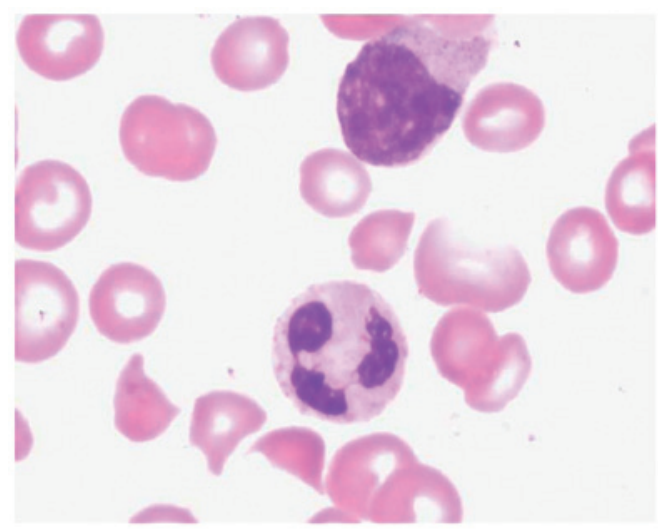

Figure 3. Case 2 bone marrow smear. Bone marrow was stained with Wright Giemsa (magnification, x1,000) demonstrating the presence of abnormally formed erythrocytes.

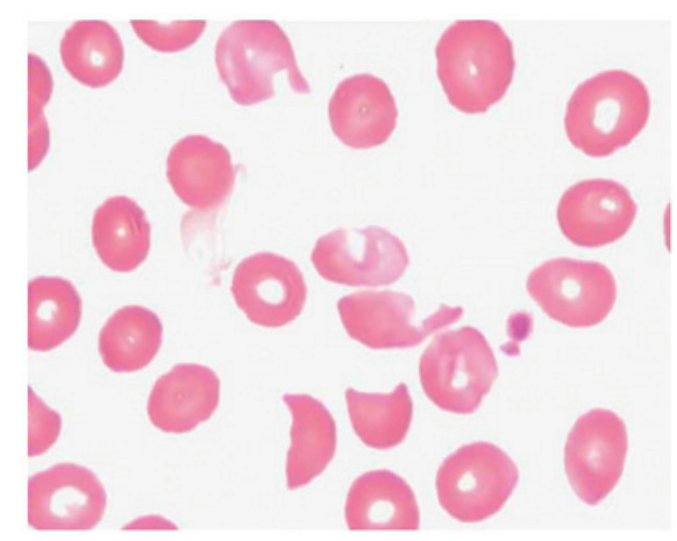

Figure 4. Case 2 peripheral blood smear. Peripheral blood was stained with Wright Giemsa (magnification, x1,000) demonstrating the presence of abnormally formed erythrocytes.

Case 2. A 35-year-old woman was admitted to the Liaocheng People's Hospital on September 18, 2013 with a 7 day history of skin petechiae and ecchymoses. A physical examination revealed that the patient had a body temperature of $37.5^{\circ} \mathrm{C}$, a heart rate of 80 beats/min, a respiratory rate of 18 breaths/min and a blood pressure of 120/80 $\mathrm{mmHg}$. In addition, the patient 
exhibited clear consciousness, appeared anemic and had light yellowish skin and scattered petechiae and bleeding sites on the mucosal membrane. A blood test on the date of admission recorded the following: Total bilirubin, $74 \mu \mathrm{mol} / 1$ and indirect bilirubin, $69 \mu \mathrm{mol} / 1$. On September 19, 2003, the blood test showed a PLT count of $7 \times 10^{9} / 1$, and the patient was intravenously treated with $16 \mathrm{U}$ PLT.

Cell morphological analyses of a bone marrow biopsy taken on September 19, 2013 were conducted. The bone marrow was aspirated from the posterior superior iliac spine, and $2 \mathrm{ml}$ bone marrow was obtained. Giemsa staining was performed on the bone marrow, and detected abnormally formed erythrocytes, indicative of TTP, reduced hyperplasia, two megakaryocytes, which were indicative of normocytic anemia, and a reduced numbers of platelets (Figs. 3 and 4). The reticulocyte count was $10.99 \%$ and the Coombs test was negative, indicating no autoimmune hemolysis. An intravenous drip administering $200 \mathrm{mg}$ methylprednisolone anti-inflammatory treatment once daily was initiated on September 20 since the patient was positive for anti-Smith $(++$, diluted $>1: 320)$ and anti-Sjögren's-syndrome-related antigen A autoantibodies (+++, diluted >1:640) detected using an IMTEC-ANA-LIA Profile (HUMAN Diagnostics, Wiesbaden, Germany), and was suspected to have connective tissue disease, systemic lupus erythematosus and possible secondary Evans syndrome.

The patient twice presented with paroxysmal numbness and speech disorder on September 22. Although the condition eased within 2 min without treatment, there was subsequent impairment in cognition and speech. On September 23, 2003 , the blood test showed a PLT count of $8 \times 10^{9} / 1$ and Hb levels of $3.4 \mathrm{~g} / 1$, and the patient was treated with $16 \mathrm{U}$ PLT and $6 \mathrm{U}$ washed red blood cells via intravenous injection. The dose of methylprednisolone was increased to $500 \mathrm{mg}$ once daily, and $20 \%$ mannitol was administered at $150 \mathrm{ml}$ every $6 \mathrm{~h}$ in order to dehydrate the patient and reduce intracranial pressure. On September 24, the patient exhibited numbness in the left-side limbs, slow responses and drooping of the mouth on the left side, with blood indices comprising an $\mathrm{Hb}$ level of $54 \mathrm{~g} / \mathrm{l}$ and PLT count of $10 \times 10^{9} / 1$. However, acute craniocerebral CT was unable to detect any apparent abnormality. The patient was diagnosed with connective tissue disease and systemic lupus erythematosus, with TTP as a complication. Subsequently, the methylprednisolone dosage was reduced to $240 \mathrm{mg}$ once daily and the patient was transferred to the ICU for PE treatment by MPS (blood flow, $120 \mathrm{ml} / \mathrm{min}$; plasma extraction rate, $24 \mathrm{ml} / \mathrm{min} ; 2,000 \mathrm{ml}$ plasma was replaced) once daily, with the consent of the family.

On September 26, the patient regained consciousness but was exhausted. On September 27, the methylprednisolone dosage was reduced to $120 \mathrm{mg}$ once daily after a blood test detected a Hb level of $3.4 \mathrm{~g} / \mathrm{dl}$ and a PLT count of $33 \times 10^{9} / 1$. On October 1, blood tests revealed a Hb level of $9.7 \mathrm{~g} / \mathrm{dl}$ and a PLT count of $125 \times 10^{9} / 1$. PE was performed once every 2 days from October 2 and the methylprednisolone dosage was reduced to $60 \mathrm{mg}$ once daily. On October 8, PE treatment was ceased and was replaced with orally administered $30 \mathrm{mg}$ prednisone three times a day following a blood test that revealed a $\mathrm{Hb}$ level of $10.3 \mathrm{~g} / \mathrm{dl}$ and a PLT count of $138 \times 10^{9} / 1$.

On October 14, a B-mode ultrasound indicated thrombosis formation around the right femoral vein catheter. In addition, the patient appeared to have recovered and was clearly conscious, with liver function and blood coagulation functions restored to within their normal ranges. The patient was discharged on October 23, 2013. At the follow-up on November 25, 2013 the patient exhibited no reoccurrence with Hb levels of $13 \mathrm{~g} / \mathrm{dl}$ and PLT counts of $183 \times 10^{9} / 1$.

\section{Discussion}

At present, PE is a very important treatment for TTP, and the mechanisms underlying its therapeutic effects include: i) Scavenging abnormal vWF multimers released by endothelial cells, ii) scavenging a disintegrin and metalloproteinase with thrombospondin motifs-like 3 (ADAMTSI3) autoantibodies in the body, iii) supplementing the $\mathrm{vWF}$ lyase ADAMTS13, and restoring the normal degradation of circulating $\mathrm{vWF}$, iv) replenishing prostacyclin I in plasma, v) scavenging abnormal antibodies in plasma, vi) scavenging various cytokines that damage endothelial cells and activate platelets, and vii) scavenging circulating inflammatory factors, including tumor necrosis factor- $\alpha$, interleukin (IL)- 6 and IL- 8 to prevent MODS (13). Patients with TTP rarely exhibit all the typical 5 symptoms of TTP, which comprises fever, changes in the nervous system, kidney damage, microvascular hemolytic anemia and consumptive reduction of platelet aggregation (14). Therefore, PE therapy should be administered as soon as possible following the detection of significant reductions in platelet numbers and microvascular hemolytic anemia, without any other clear etiology. Due to financial constraints, the family of case 1 agreed to PE therapy only on day 5 following hospital admission, on which day the patient experienced continuous convulsions and was comatose, requiring mechanical ventilation for respiratory failure. Case 2 showed disturbances in consciousness on day 5 following hospital admission, and underwent PE in the ICU after being diagnosed with TTP on day 7. In these two patients, $\mathrm{PE}$ therapy was initiated at a late stage; however, both regained consciousness after three PE sessions. Mechanical ventilation and tracheal intubation were removed from case 1 after two PE treatments, and after seven PE sessions, the patient exhibited a markedly improved mental status, was able to answer simple questions, and had an increased PLT of $41 \times 10^{9} / 1$. After $12 \mathrm{PE}$ sessions, the patient was stable and was transferred from the Hematology Unit to the dialysis room to receive PE treatment once every 2 days for a total of $23 \mathrm{PE}$ treatments. Case 2 stopped having seizures after regaining consciousness and her platelet counts were restored to within the normal range after 10 PE sessions. Case 2 was transferred from the Hematology Unit to the dialysis room to receive PE treatment once every 2 days, prior to discharge from the hospital. Both patients were in a serious condition upon admission and thus their PE treatments were initiated in the ICU.

The ICU at the Liaocheng People's Hospital initially performed blood purification procedures in 2004 to treat patients with systemic inflammatory response syndrome, sepsis, all types of intoxication and MODS. PE was performed for the first time in our ICU for these two patients. It was found to be effective, convenient and fast since the ICU staff were able to complete the procedure independently without moving the patients. PE is a type of blood 
purification technique, which involves extraction of plasma using the MPS technique and its replacement with an equal volume of fresh frozen plasma or human blood albumin, in order to scavenge various metabolic toxins and pathogenic factors (15). PE filters differ from filters used in other blood purification techniques; PE filters comprise hollow fiber-type separators, prepared from cellulose acetate, poly(methyl methacrylate) or polysulfone membranes (16). The present study employed a hollow-fiber-type separator with a polysulfone membrane, and achieved good efficacy in these two cases without reoccurrence within 1 month of discharge from the hospital.

In conclusion, the present study successfully treated two patients with severe TTP using PE, and in doing so developed a strategy for the treatment of patients with severe conditions who cannot be treated in the dialysis room. Furthermore, this provides a novel concept for the treatment of patients with TTP, rheumatic autoimmune disease, Guillain-Barre syndrome and acute myelitis. However, special attention should be given to complications associated with the long-term use of PE, including systemic infection, partial or total blockage of catheters, low blood pressure, venous thrombosis formation, and bleeding or pneumothorax resulting from catheterization. The two patients in the present study exhibited venous thrombosis in their lower limbs. Case 1 showed venous thrombosis earlier and predominantly within muscular veins instead of in the catheterized femoral vein; therefore the venous thrombosis in the patient was likely associated with the TTP. Conversely, case 2 exhibited venous thrombosis 23 days following catheterization in the femoral vein around the catheter. The patient was relatively stable at that time with platelet counts within the normal range, thus suggesting that the venous thrombosis was likely due to long-term catheterization.

\section{References}

1. Allford SL, Hunt BJ, Rose P and Machin SJ; Haemostasis and Thrombosis Task Force, British Committee for Standards in Haematology: Guidelines on the diagnosis and management of the thrombotic microangiopathic haemolytic anaemias. Br J Haematol 120: 556-573, 2003.
2. Coppo P1, Wolf M, Veyradier A, Bussel A, Malot S, Millot GA, Daubin C, Bordessoule D, Pène F, Mira JP, et al: Prognostic value of inhibitory anti-ADAMTS13 antibodies in adult-acquired thrombotic thrombocytopenic purpura. Br J Haematol 132: 66-74, 2006.

3. Rock GA: Management of thrombotic thrombocytopenic purpura. Br J Haematol 109: 496-507, 2000.

4. O'Connor NT, O'Shea MJ and Hill LF: Vincristine for thrombotic thrombocytopenic purpura. Lancet 340: 490, 1992.

5. Durand JM, Lefevre P, Kaplanski G, Telle H and Soubeyrand J: Vincristine for thrombotic thrombocytopenic purpura. Lancet 340: 977-978, 1992.

6. Durand JM, Lefevre P, Kaplanski G and Soubeyrand J: Ineffectiveness of high-dose intravenous gammaglobulin infusion in thrombotic thrombocytopenic purpura. Am J Hematol 42: 234, 1993.

7. Udvardy M and Rak K: Cyclophosphamide for chronic relapsing thrombotic thrombocytopenic purpura. Lancet 336: 1508-1509, 1990.

8. Schneppenheim R and Budde U: von Willebrand factor: The complex molecular genetics of a multidomain and multifunctional protein. J Thromb Haemost 9 (Suppl 1): 209-215, 2011.

9. Motto DG, Chauhan AK, Zhu G, Homeister J, Lamb CB, Desch KC, Zhang W, Tsai HM, Wagner DD and Ginsburg D: Shigatoxin triggers thrombotic thrombocytopenic purpura in genetically susceptible ADAMTS13-deficient mice. J Clin Invest 115: 2752-2761, 2005.

10. Zheng XL, Kaufman RM, Goodnough LT and Sadler JE: Effect of plasma exchange on plasma ADAMTS13 metalloprotease activity, inhibitor level and clinical outcome in patients with idiopathic and nonidiopathic thrombotic thrombocytopenic purpura. Blood 103: 4043-4049, 2004.

11. Gadisseur A, Hermans C, Berneman Z, Schroyens W, Deckmyn H and Michiels JJ: Laboratory diagnosis and molecular classification of von Willebrand disease. Acta Haematol 121: 71-84, 2009.

12. Bandarenko N and Brecher ME: United States Thrombotic Thrombocytopenic Purpura Apheresis Study Group (US TTP ASG): Multicenter survey and retrospective analysis of current efficacy of therapeutic plasma exchange. J Clin Apher 13: 133-141, 1998.

13. Yao LQ, Jin ZC, Ji MS, Xia CY, Yu ZX, Liu J, Hu XL and Yan J: Effect of continuous renal replacement therapy started at different time on patients with multiple organ dysfunction syndrome. Zhonghua Yi Xue Za Zhi 91: 1663-1667, 2011 (In Chinese).

14. Sadler JE, Moake JL, Miyata T and George JN: Recent advances in thrombotic thrombocytopenic purpura. Hematology Am Soc Hematol Educ Program 2004: 407-423.

15. Paton E and Baldwin IC: Plasma exchange in the intensive care unit: A 10 year retrospective audit. Aust Crit Care 27: 139-44, 2014.

16. Maruyama Y, Yoshida H, Uchino S, Yokoyama K, Yamamoto H, Takinami $\mathrm{M}$ and Hosoya T: Nafamostat mesilate as an anticoagulant during continuous veno-venous hemodialysis: A three-year retrospective cohort study. Int J Artif Organs 34: 571-576, 2011. 